\title{
Effect of dihydroarteminin combined with siRNA targeting Notch1 on Notch1/c-Myc signaling in T-cell lymphoma cells
}

\author{
LANFEN HUO $^{1 *}$, WENWEN WEI ${ }^{1 *}$, SHAOLING WU ${ }^{1}$, XINDONG ZHAO $^{2}$, \\ CHUNTING ZHAO ${ }^{1}$, HONGGUO ZHAO ${ }^{1}$ and LINGJIE SUN ${ }^{1}$ \\ ${ }^{1}$ Department of Hematology, Affiliated Hospital, Qingdao University, Qingdao, Shandong 266003; ${ }^{2}$ Department of \\ Hematology, School of Medicine, Qingdao University, Qingdao, Shandong 266021, P.R. China
}

Received September 12, 2017; Accepted January 16, 2018

DOI: $10.3892 / \mathrm{etm} .2018 .5784$

\begin{abstract}
The effectiveness of therapy combining dihydroartemisinin (DHA) and small interfering RNA targeting Notch1 (siNotch1) in T-cell lymphoma remains unknown. The present study explored the potential and possible mechanisms of combined dihydroarteminin, and siNotch1 therapy for T-cell lymphoma. It was demonstrated that the viability rates of siRNA-DHA-treated cells was significantly suppressed in comparison with those in control cells, control siRNA cells, siRNA-treated cells and DHA-treated cells $(\mathrm{P}<0.01)$. Additionally, there was a significant increase in cell apoptosis of siRNA-DHA-treated cells in comparison with those of control cells, control siRNA cells, siRNA-treated cells, DHA-treated cells $(\mathrm{P}<0.05)$. Furthermore, Notch1 and $\mathrm{c}-\mathrm{Myc}$ mRNA and protein expression were decreased in siRNA-DHA-treated cells $(\mathrm{P}<0.05)$. The present study demonstrated that DHA combined with siNotch1 is able to suppress proliferation and promote apoptosis, and downregulate the expression of Notch1 and c-Myc mRNA and protein in T-cell lymphoma cells. Targeting Notch1/c-Myc signaling with siRNA and
\end{abstract}

Correspondence to: Professor Shaoling Wu, Department of Hematology, Affiliated Hospital, Qingdao University, 16 Jiangsu Road, Qingdao, Shandong 266003, P.R. China

E-mail: qdwushaoling@163.com

Professor Xindong Zhao, Department of Hematology, School of Medicine, Qingdao University, 203B Room, Boya Building, 308 Ningxia Road, Qingdao, Shandong 266021, P.R. China

E-mail: qdzhaoxindong@163.com

*Contributed equally

Abbreviations: DHA, dihydroartemisinin; siNotch1, siRNA targeting Notch1; CCK-8, Cell Counting Kit-8; RT-PCR, reverse transcription PCR; RNAi, RNA interference; DMSO, dimethyl sulfoxide; PBS, phosphate-buffered saline; MOI, multiplicity of infection

Key words: siRNA, Notch1 signaling, dihydroartemisinin, c-Myc, T-cell lymphoma
DHA may represent a novel strategy for treating human T-cell lymphoma.

\section{Introduction}

Lymphoma is a malignant tumor of the hematological system that has high histological and genetic heterogeneity. Lymphoma is classified into B-cell or T-cell lymphoma, depending on the pathological type. Several approaches including radiotherapy, chemotherapy, and hematopoietic stem cell transplantation have shown some efficacy in treating T-cell lymphoma; however, the prognosis and survival of patients with T-cell lymphoma remain poor (1). RNA interference (RNAi) has recently emerged as a potential strategy for targeted therapy, and the antitumor effects of several RNAi strategies in tumor cells are currently under exploration. However, few studies have investigated the therapeutic effect of RNAi on T-cell lymphoma.

Notch1 signaling regulates self-renewal, proliferation, differentiation, and apoptosis in T-cells $(2,3)$. The c-Myc protein, which is encoded by a gene that is a downstream target of Notch1, regulates protein stabilization, promotes cell division and accelerates cell entry to $\mathrm{S}$ phase from $\mathrm{G0} / \mathrm{G} 1$ phase. Previous studies showed that mutations in the Notch1 gene result in sustained activation of Notch1 signaling, eventually leading to the occurrence of $\mathrm{T}$ cell tumors in mice (4). In addition, c-Myc promotes cell proliferation and inhibits apoptosis in many tumor cells (5-7). Mutation of the Notch1 gene may lead to T-cell lymphoblastic leukemia by directly increasing c-Myc expression. Furthermore, a previous study showed that Notch1 and c-Myc cooperate in the development of T-cell lymphoblastic leukemia in vivo, and Notch1 may promote the growth of leukemic cells by maintaining c-Myc mRNA levels (8). Other studies found that the inhibition of Notch1 signaling with small molecule inhibitors in mouse leukemic cells led to an increase apoptosis and decrease of c-Myc expression (4). Together these studies suggest that the Notch1/c-Myc signaling pathway contributes to T-cell lymphoma oncogenesis. Previous research has suggested that siRNA targeting Notch1 (siNotch1) could significantly suppress proliferation and promote apoptosis in lymphoma cells, and siNotch1 also exhibited anti-cancer activity in non-small cell lung cancer and malignant melanoma via its effects on the Notch1 signaling pathway (9-11). 
Together these data suggest that siNotch1 may be an effective anti-cancer agent in T-cell lymphoma.

Dihydroartemisinin (DHA), which is derived from the traditional Chinese medical herb Artemisia annua L., is widely used as an antimalarial drug (12) and has a cytotoxic effect on T-cell lymphoma cells (13). Pure DHA can block endothelial cell proliferation via effects on the ERK signaling pathway (14). Additionally, histone deacetylase inhibitors combined with DHA have been shown to activate caspase-3 and enhance the anticancer effect of the ERK signaling pathway in liver tumors (15). Co-treatment of DHA with gemcitabine has been demonstrated to exhibit a therapeutic effect in a pancreatic cancer model, via a proposed mechanism involving NF- $\mathrm{\kappa B}$ inactivation (16).

To improve treatment outcomes for T-cell lymphoma, here we carried out research to explore the possible mechanisms of combined DHA and siNotch1 therapy for T-cell lymphoma using Jurkat cells, and we also explored the involvement of the Notch1/c-Myc signaling pathway in mediating any antineoplastic effect of this treatment. We hope that this study may provide the basis for a novel, efficient, and safe treatment for T-cell lymphoma.

\section{Materials and methods}

Cell culture. Jurkat cells (a T-cell lymphoma cell line) were purchased from the Chinese Academy of Sciences Cell Bank. Cells were grown in RPMI-1640 medium (HyClone, Logan, UT, USA) in 10\% FBS (Gibco, Carlsbad, CA, USA), penicillin and streptomycin, and were cultured at $37^{\circ} \mathrm{C}$ in $5 \% \mathrm{CO}_{2}$ in a humidified incubator. Jurkat cells in the logarithmic growth phase were used for all experiments.

Detection of Notch1 DNA mutations. Jurkat cell DNA was isolated according to the manufacturer's instructions (Thermo Fisher Scientific, Inc., Waltham, MA, USA). Notch1 DNA sequence mutations in exons 27 and 34 (Fig. 1) were detected by MapBioo (Shanghai, China).

Notch siRNA. The siNotch1 sequences (siRNA I and II) were designed and synthesized by Genechem (Shanghai, China). Jurkat cells were seeded in 6 -well plates $\left(2 \times 10^{5}\right.$ cells $/$ well in $1 \mathrm{ml}$ culture medium) and divided into three groups: Then control siRNA cells were transfected with control siRNA at multiplicity of infection $(\mathrm{MOI})=80$ (lentiviral vector titer $1 \times 10^{9}$; sequence, TCTCCGAACGTGTCACGT), siRNA I-treated cells were transfected with siRNA I $(\mathrm{MOI}=80$, lentiviral

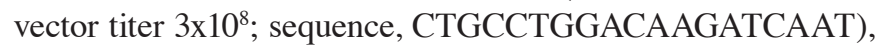
and siRNA II-treated cells were transfected with siRNA II (MOI $=80$, lentiviral vector titer $3 \times 10^{8}$; sequence, TGCCAA ATGCCTGCCAGAA). Last Fluorescence in transfected cells was observed by laser scanning confocal microscope after $72 \mathrm{~h}$. Preliminary experiments indicated that siRNA I had a higher transfection rate than siRNA II, so siRNA I was used for the subsequent experiments. Since the lentiviral vector carries the anti-puromycin gene, stably transfected Jurkat cells were selected using puromycin $(2 \mu \mathrm{g} / \mathrm{ml})$.

Determination of optimal DHA concentration. DHA (Chunyou Biological Technology Co., Ltd., Shanghai,
Table I. Gene-specific primer sequences for reverse transcription quantitative-polymerase chain reaction.

\begin{tabular}{lc}
\hline Gene & Sequence \\
\hline GAPDH & 5'-GATGACCTTGCCCACAGCCT-3' \\
& 5'-ATCTCTGCCCCCTCTGCTGA-3' \\
Notch1 & 5'-CCAGTTTGAATGGTCAATGC-3' \\
& 5'-AGAGGGTTGTATTGGTTCGG-3' \\
c-Myc & 5'-CTACCCTCTCAACGACAGCA-3' \\
& 5'-AGAGCAGAGAATCCGAGGAC-3' \\
Caspase-3 & 5'-TAAATGAATGGGCTGAGCTG-3' \\
& 5'-ATGGAGAAATGGGCTGTAGG-3'
\end{tabular}

China) was dissolved in dimethyl sulfoxide (Sigma-Aldrich; Merck KGaA, Darmstadt, Germany) to form an $8 \mathrm{mM}$ stock solution and stored at $-20^{\circ} \mathrm{C}$.

Jurkat cells $\left(8 \times 10^{3}\right.$ cells/well in $100 \mu \mathrm{l}$ medium) were seeded in 96-well plates, and various concentrations of DHA $(0,2.5,5,10,20$, or $40 \mu \mathrm{M})$ were added. After 24,48 , or $72 \mathrm{~h}$, $10 \mu \mathrm{l}$ CCK-8 reagent was added and the cells were incubated for $2 \mathrm{~h}$. Cell viability was then assessed by CCK-8 assay (10 $\mu \mathrm{l}$ reagent/well; Dojindo Molecular Technologies, Inc., Kyushu, Japan). The optimal DHA treatment was determined as $20 \mu \mathrm{M}$ for $24 \mathrm{~h}$.

Cell viability assay. Five groups were included in the experiments: untreated control cells, control siRNA cells, siRNA I-treated cells, DHA-treated cells, and siRNA-DHA-treated cells. Jurkat cells $\left(8 \times 10^{3}\right.$ cells/well in $100 \mu 1$ medium) were seeded in 96-well plates. DHA $(20 \mu \mathrm{M})$ was added, and the cells were cultured for $24 \mathrm{~h}$. Cell viability was subsequently assessed by CCK- 8 assay. A microplate absorbance reader (Tecan Group Ltd., Männedorf, Switzerland) was used to measure absorbance at $450 \mathrm{~nm}$. Each experiment was repeated three times.

Cell apoptosis assay. Jurkat cells were seeded in 6-well plates ( $2 \times 10^{5}$ cells/well in $1 \mathrm{ml}$ culture medium). After $24 \mathrm{~h}$, cells were collected and washed once in phosphate-buffered saline (PBS) and once in binding buffer, and then resuspended in binding buffer. Fluorochrome-conjugated Annexin V (5 $\mu \mathrm{l})$ was added, and the cells were incubated for $15 \mathrm{~min}$ at room temperature. Next, $5 \mu \mathrm{l}$ of propidium iodide staining solution was added. Samples were analyzed by flow cytometry (BD FACSCalibur; BD Biosciences, Franklin Lakes, NJ, USA).

$R T$ - $q P C R$. Total RNA was extracted using TRIzol reagent (Takara, Tokyo, Japan) and reverse-transcribed using a Roche kit (Roche Diagnostics GmbH, Mannheim, Germany) according to the manufacturer's instructions. RNA quality and quantity were assessed by spectrophotometry (Dynamica Scientific Ltd., Milton Keynes, UK). Gene-specific primers were synthesized by Sangon Biotech (Shanghai, China); sequences are provided in Table I. The RT-qPCR program used was as follows: Preincubation at $95^{\circ} \mathrm{C}$ for $600 \mathrm{sec} ; 40$ cycles of amplification, with initial denaturation at $95^{\circ} \mathrm{C}$ for $10 \mathrm{sec}, 60^{\circ} \mathrm{C}$ 

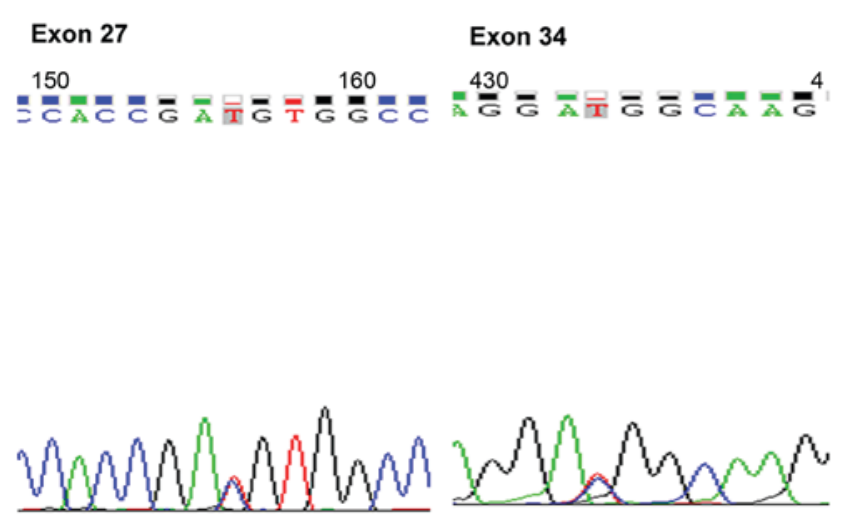

Figure 1. DNA mutations of Notch1 in exon 27,34 by DNA sequencing. DNA mutations of Notch1 in exon 27, 34 as follows: There is a point mutation of 156th in exon 27 ( $\mathrm{T}$ instead of $\mathrm{C}$ ) and a point mutation of 433rd in exon 34 (C mutation to T).

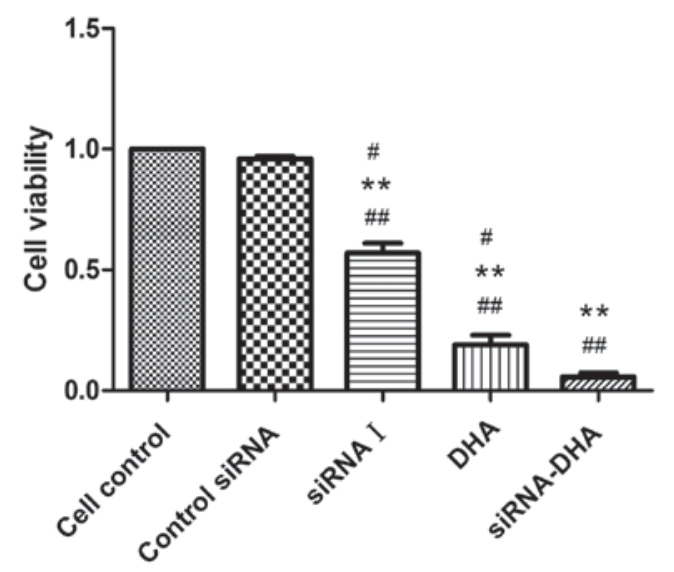

Figure 2. DHA combined with siNotch1 decreased Jurkat cell viability Jurkat cells were treated with DHA alone or siNotch1 alone, or combined therapy. ${ }^{\# \#} \mathrm{P}<0.01$, compared with cell control; ${ }^{* *} \mathrm{P}<0.01$, compared with control siRNA cells; and " $\mathrm{P}<0.01$, compared with siRNA-DHA-treated cells.

for $20 \mathrm{sec}$, and then $72^{\circ} \mathrm{C}$ for $20 \mathrm{sec}$; melting was performed at $95^{\circ} \mathrm{C}$ for $10 \mathrm{sec}, 65^{\circ} \mathrm{C}$ for $60 \mathrm{sec}$ and $97^{\circ} \mathrm{C}$ for $1 \mathrm{sec}$. The $2^{-\Delta \Delta \mathrm{Cq}}$ method (17) was used to calculate relative expression of Notch1, c-Myc, and caspase-3 mRNA.

Western blot analysis. Jurkat cells were collected and protein was extracted using Radio Immunoprecipitation Assay and phenylmethanesulfonyl fluoride (Beyotime, Shanghai, China). Protein were analyzed by spectrophotometry. Equivalent amounts of protein with adjusted concentration were separated by SDS-PAGE (10\%) and transferred to polyvinylidene difluoride membranes. Membranes were blocked with $5 \%$ fat-free milk and incubated with primary antibodies $(1: 1,000)$ for $2 \mathrm{~h}$, followed by secondary antibody $(1: 2,000)$ (both from Abcam, Cambridge, UK) for $1 \mathrm{~h}$ at room temperature. $\beta$-actin was used as a loading control. An ECL chemiluminescence kit (Thermo Fisher Scientific, Inc.) was used to visualize the signals. Images were analyzed using imaging software (Vilber Lourmat, Marne-la-Vallée, France).

Statistical analysis. All data were analyzed by one-way ANOVA using Graphpad Prism 5.0. All results are expressed
A

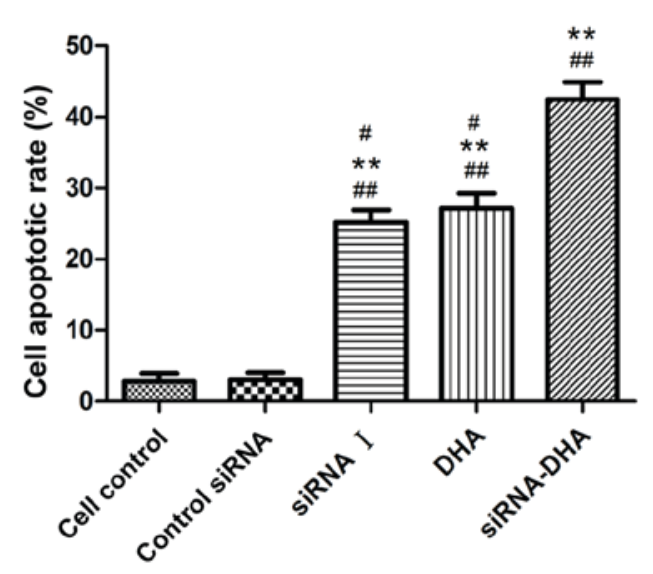

B

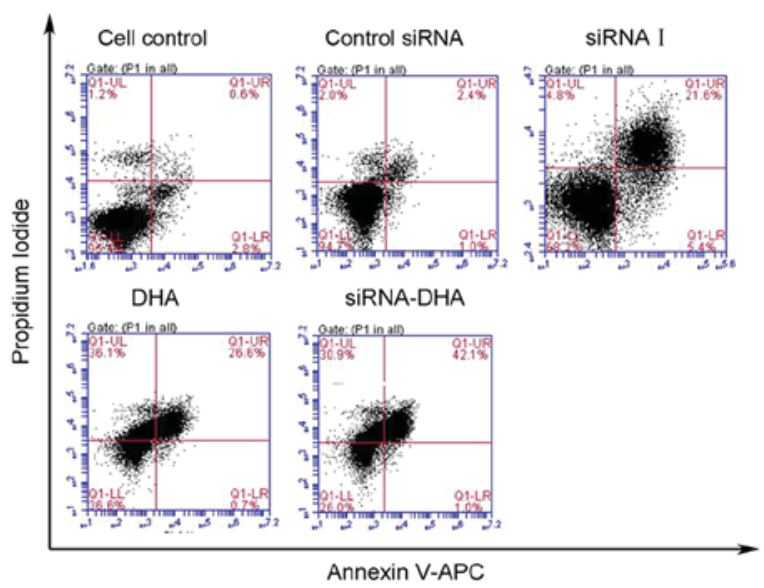

Figure 3. DHA combined with siNotch1 facilitated Jurkat cells apoptosis. (A) DHA combined with siNotch1 further induced Jurkat cells apoptosis. ${ }^{\# \#} \mathrm{P}<0.01$, compared with cell control; ${ }^{* *} \mathrm{P}<0.01$, compared with control siRNA cells; " $\mathrm{P}<0.01$, compared with siRNA-DHA-treated cells. (B) Jurkat cells apoptosis was measured by Annexin $\mathrm{V}$ and propidium iodide staining solution using flow cytometry.

as mean \pm standard deviation (mean $\pm \mathrm{SD}$ ). The differences between groups were analyzed using LSD-t. $\mathrm{P}<0.05$ was considered to indicate a statistically significant difference.

\section{Results}

DHA combined with siNotch1 decreases Jurkat cell viability. We first evaluated the viability of Jurkat cells in response to Notch siRNA, DHA $(20 \mu \mathrm{M})$ or the combined treatment for $24 \mathrm{~h}$ using CCK-8 assays (Fig. 2). Compared with the viability of control siRNA cells (95.90\%), the cell viabilities for siRNA I-, DHA- and siRNA-DHA-treated cells were 57.25, 19.12, and $5.66 \%$, respectively. These results demonstrate that DHA combined with siNotch1 showed enhanced reduction of cell viability compared with compared with either treatment alone.

DHA combined with siNotchl promotes Jurkat cell apoptosis. DHA combined with siRNA treatment significantly enhanced Jurkat cell apoptosis (Fig. 3). The Jurkat cell apoptosis rates for cell control, and control siRNA cells, siRNA I-, DHA-, 


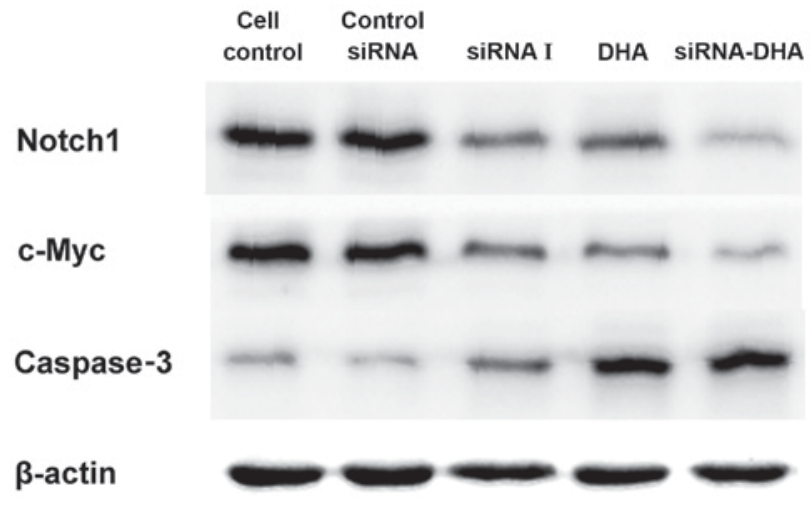

Figure 4. The results of western blot analysis for Notch1, c-Myc and caspase-3. DHA combined with siRNA further downregulated Notch1 and c-Myc protein and upregulated caspase-3 protein.

and siRNA-DHA-treated cells were: $2.83,3.00,25.17,27.17$, and $42.47 \%$, respectively. DHA combined with siNotch 1 promoted higher Jurkat cell apoptosis $(42.47 \%)$ compared with siNotch- or DHA-treated cells $(\mathrm{P}<0.01, \mathrm{P}<0.01)$. These results demonstrate that DHA combined with siNotch1 promoted Jurkat cell apoptosis.

DHA combined with siNotch1 inhibits Notchl mRNA and protein expression in Jurkat cells. We evaluated Notch1 mRNA and protein levels in the control siRNA cells, siRNA I-, DHA-, and siRNA-DHA-treated cells groups (Figs. 4 and 5). Notch1 mRNA expression in cell control, and in control siRNA cells, siRNA I-, DHA-, and siRNA-DHA-treated cells were $1.00 \pm 0.04,0.99 \pm 0.13,0.58 \pm 0.09,0.36 \pm 0.05$, and $0.16 \pm 0.05$, respectively (Fig. 5A). Compared with cell control, control siRNA, siRNA I-, DHA-, and siRNA-DHA treatment decreased expression of Notch1 mRNA by 1.12, 41.97, 63.86 , and $84.23 \%$, respectively. Significant differences were found between the siRNA-DHA- and siRNA I-treated cells $(\mathrm{P}<0.01)$, and between the siRNA-DHA- and DHA-treated cells $(\mathrm{P}<0.05)$.

Fig. 5B shows the relative expression of Notch1 protein. Notch1 protein expression in cell control, and in control siRNA cells, siRNA I-, DHA-, and siRNA-DHA-treated cells were $1.09 \pm 0.03,1.04 \pm 0.01,0.76 \pm 0.01,0.67 \pm 0.02$, and $0.47 \pm 0.07$, respectively. Of all the treatment conditions tested, the Notch1 protein level was reduced the most by treatment with DHA combined with siNotch1.

DHA combined with siNotch1 inhibits $c-M y c m R N A$ and protein expression in Jurkat cells. Expression of c-Myc mRNA and protein in Jurkat cells treated with DHA combined with siNotch1 (Figs. 4 and 6). Treatment with siRNA-DHA, DHA and siRNA I decreased expression of c-Myc mRNA by 96.86 , 92.33 , and $72.91 \%$, respectively, compared with expression in cell control (Fig. 6A).

c-Myc protein expression levels followed a similar pattern to c-Myc mRNA expression levels. Treatment with siRNA-DHA, siRNA I, and DHA decreased expression of c-Myc protein by 85.19, 34.81, and $60.12 \%$, respectively, compared with expression in cell control $(\mathrm{P}<0.01, \mathrm{P}<0.05, \mathrm{P}<0.05$; Fig. $6 \mathrm{~B})$. Of all the treatment conditions tested, $\mathrm{c}-\mathrm{Myc}$ mRNA and protein

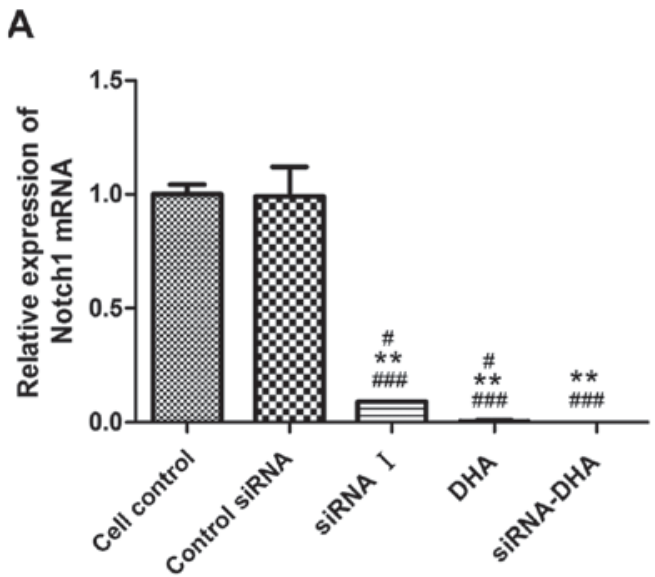

B

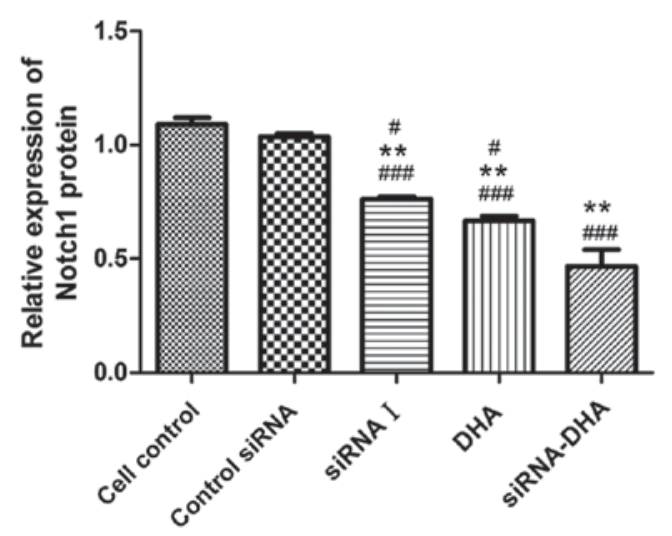

Figure 5. DHA combined with siNotch1 inhibited the expression of Notch1 mRNA and protein in Jurkat cells. (A) DHA combined with siNotch1 downregulated the expression of Notch1 mRNA in Jurkat cells. ${ }^{\# \#} \mathrm{P}<0.01$, compared with cell control; ${ }^{* *} \mathrm{P}<0.01$, compared with control siRNA cells; ${ }^{\#} \mathrm{P}<0.05$, compared with siRNA-DHA-treated cells. (B) The expression of Notch1 protein in Jurkat cells was also decreased. ${ }^{\# \# ~} \mathrm{P}<0.01$, compared with cell control; ${ }^{* *} \mathrm{P}<0.01$, compared with control siRNA cells; and ${ }^{\#} \mathrm{P}<0.01$, compared with siRNA-DHA-treated cells.

expression were reduced the most by treatment with DHA combined with siNotch1.

DHA combined with siNotch1 promotes caspase-3 mRNA and protein expression in Jurkat cells. caspase-3 mRNA and protein expression were promoted in Jurkat cells treated with DHA combined with siNotch1 (Figs. 4 and 7). Treatment with siRNA-DHA, DHA, and siRNA I increased the expression of caspase 3 mRNA by $99.36,98.21$, and $96.66 \%$, respectively, compared with expression in cell control (Fig. 7A). siRNA-DHA treatment promoted caspase-3 mRNA expression more strongly than siRNA I or DHA treatment alone $(\mathrm{P}<0.01, \mathrm{P}<0.01)$.

siRNA-DHA, DHA, and siRNA I treatment increased caspase-3 protein levels by $46.79,38.52$, and $33.60 \%$, respectively, compared with levels in cell control (Fig. 7B). There were significant differences in caspase-3 protein levels between siRNA-DHA-treated cells and DHA-treated or siRNA I-treated cells $(\mathrm{P}<0.01, \mathrm{P}<0.01)$. Treatment of Jurkat cells with combined DHA and siNotch1 promoted caspase-3 protein expression to a greater extent than treatment with either alone. 
A

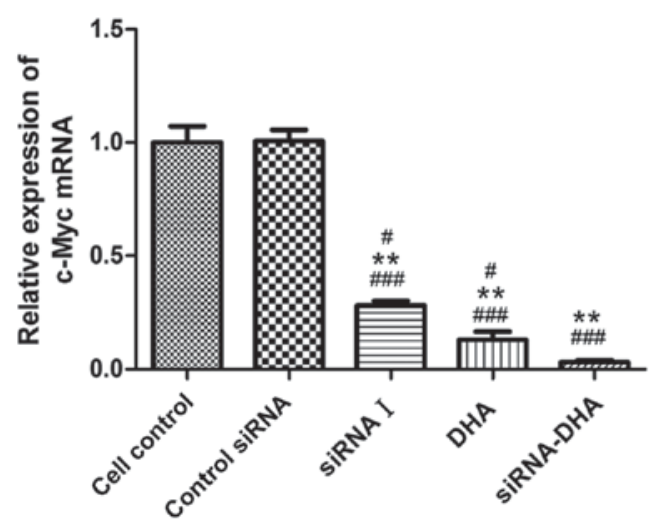

B

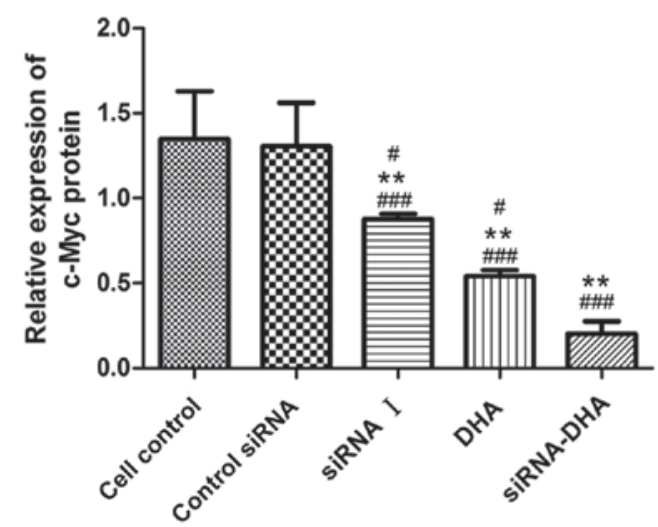

Figure 6. DHA combined with siNotch1 inhibited c-Myc mRNA and protein expression in Jurkat cells. (A) Treatment of Jurkat cells with dihydroarteminin and siNotch1 inhibited c-Myc mRNA expression. ${ }^{\# \# \#} \mathrm{P}<0.01$, compared with cell control; ${ }^{* * *} \mathrm{P}<0.01$, compared with siRNA-treated cells; and ${ }^{\#} \mathrm{P}<0.05$, compared with siRNA-DHA-treated cells. (B) c-Myc protein expression in Jurkat cells was decreased. ${ }^{\# \# \#} \mathrm{P}<0.01$, compared with cell control; ${ }^{* *} \mathrm{P}<0.05$, compared with control siRNA cells; and " $\mathrm{P}<0.05$, compared with siRNA-DHA-treated cells.

A

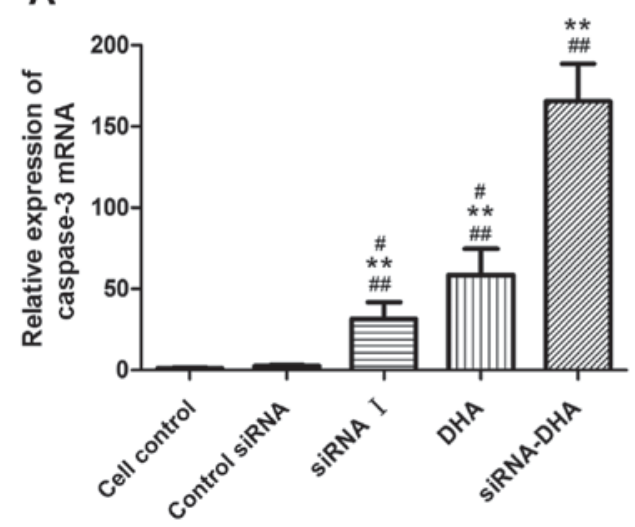

B

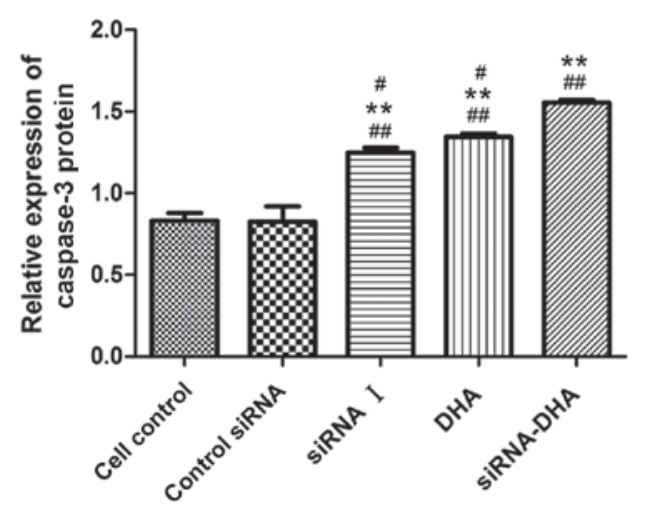

Figure 7. DHA combined with siNotch1 fortified caspase-3 mRNA and protein expression in Jurkat cells. (A) DHA combined with siNotch1 enhanced the expression of caspase-3 mRNA in Jurkat cells. ${ }^{\# \#} \mathrm{P}<0.05$, compared with cell control; ${ }^{* *} \mathrm{P}<0.05$, compared with siRNA control cells; ${ }^{*} \mathrm{P}<0.01$, compared with siRNA-DHA-treated cells. (B) Treatment of Jurkat cells with DHA and siNotch1 fortified caspase-3 protein expression. ${ }^{\# \#} \mathrm{P}<0.01$, compared with cell control; ${ }^{* *} \mathrm{P}<0.01$, compared with control siRNA cells; ${ }^{*} \mathrm{P}<0.05$, compared with siRNA-DHA-treated cells, the results presented significant difference.

\section{Discussion}

Notch1 signaling is closely related to T-cell lymphoma oncogenesis $(18,19)$. Therefore, therapy targeting Notch1 signaling may be an effective approach for treating T-cell lymphoma and we hypothesized that silencing Notch1 gene by RNAi technology in T-cell lymphoma cells may be a useful therapeutic strategy. DHA is an anti-tumor drug from traditional Chinese herbal medicine, and thus DHA combined with siNotch1 may be more effective for lymphoma therapy. The aim of the study was to determine whether the novel combination treatment of DHA with siNotch1 was effective against for T-cell lymphoma, and thus we explored the cytotoxic effects of DHA and siNotch1 alone and combined in T-cell lymphoma cells.

Jurkat cells (a T-cell lymphoma cell line) were sensitive to DHA alone in vitro (Fig. 2). Consistent with our results, previous studies showed that DHA alone exhibits antitumor activity in lung, breast, colon, and pancreatic cancer by suppressing proliferation (20). We also observed that Jurkat cells were sensitive to siNotch1 alone in vitro (Fig. 2). Previous studies also showed that targeted RNAi therapy can inhibit proliferation and invasion of human gastric carcinoma cells and human prostate cancer cells in vitro $(21,22)$. Our findings showed that DHA combined with siNotch1 reduced cell proliferation (Fig. 2) and induced cell apoptosis (Fig. 3) in Jurkat cells. Together, the results of our study indicated that DHA combined with siNotch1 was highly effective for Jurkat cells compared with DHA or siNotch1 alone. We will further study the underlying mechanisms of how DHA promote the function of siNotch and inhibit proliferation in T-cell lymphoma cells.

The cytotoxic mechanisms of DHA have been investigated in several studies, showing that dihydroarteminin enhances cell apoptosis via its effects on the ERK signaling pathway in endothelial cells (14), inactivating NF- $\mathrm{BB}$ in pancreatic cancer (16) and by upregulation of Noxa by promotion of FOXO3a expression in acute myeloid leukemia cells (23). Additionally, DHA exerts an antineoplastic effect in prostate cancer cells by increasing death receptor 5 expression (24) and induces NOXA-dependent mitochondrial cell apoptosis in melanoma cells with upregulation of cellular oxidative 
stress (25), furthermore, DHA triggers cell cycle arrest via effects on the AKT/GSK3ß/cyclin D1 pathway in A549 lung cancer cells (26). DHA could also inhibit the Wnt/ $\beta$-catenin signaling pathway in lung cancer, and the expressions of key proteins including Wnt5-a/b, LRP6 and Dvl2 in the Wnt/ $\beta$-catenin signaling pathway were significantly decreased (27). More importantly, DHA has been shown to increase reactive oxygen species generation, downregulate transferrin receptor mRNA expression and telomerase activity, upregulate NOXA protein in T-cell lymphoma cells $(13,28)$. We intend to study the mechanisms underlying the effects of DHA on NOXA signaling in T cell lymphoma cells in future. Together these results showed that DHA is a regulator of signaling pathways and gene expressions in cancer cells. Taken together, these studies, including our study, showed that DHA exhibits a therapeutic effect in cancer cells, including T-cell lymphoma cells, through regulation of signaling pathways and gene expressions.

We propose that inhibition of Notch1/c-Myc signaling is one of the mechanisms by which DHA combined with siNotch1 exert a marked antineoplastic effect on T-cell lymphoma cells: The relative expression of c-Myc mRNA and protein were markedly decreased, and as a direct downstream target gene of Notch1, c-Myc can be inhibited via the Notch1/ c-Myc signaling pathway. Our findings showed the silencing of Notch1 gene and/or DHA may downregulate the expression level of c-Myc mRNA and protein in T-cell lymphoma cells (Figs. 4 and 6). Sharma et al (8) also found that c-Myc levels clearly correlate with Notch1 activity in acute T-cell leukemia, and c-Myc could be induced in the presence of active Notch1 signaling. Active Notch1 signaling induces the overexpression of c-Myc, and c-Myc may activate many genes in cellular processes, including those involved in promoting growth and proliferation. These data indicate that Notch1 is necessary for T-cell lymphoma cell proliferation, so silencing the Notch1 gene may exhibit a therapeutic effect in T-cell lymphoma cell growth control via reducing the overexpression of c-Myc.

Our results showed that DHA combined with siNotch1 could inhibit the Notch1/c-Myc pathway in T-cell lymphoma cells in vitro. Some studies also showed that RNAi technology exerts an antineoplastic effect on cancer cells (9,21,29-31). Activation of Notch signaling promotes tumor progression by regulation of downstream target genes such as c-Myc. DHA combined with siRNA targeting Racl also induces apoptosis by inhibiting $\mathrm{NF}-\kappa \mathrm{B}$ activity in colon tumor cells (28). These observations may partly explain our results, showing that DHA combined with siNotch1 could inhibit the Notch1/c-Myc pathway in T-cell lymphoma cells.

In this study, T-cell lymphoma cell proliferation was suppressed and apoptosis was promoted following DHA combined with siNotch1. Furthermore, expression levels of Notch1 and c-Myc mRNA and protein were reduced in T-cell lymphoma cells by treatment with DHA or siNotch1 alone or in combination, and caspase- $3 \mathrm{mRNA}$ and protein expression levels were increased. We also intend to study the impact of DHA on cleavaged caspase 3 in future. These effects were greatly enhanced by combining the two treatments, which suggested that the antitumor effect of DHA combined with siNotch1 was superior to that of each agent alone. Our findings suggest that the cytotoxic effect of the combined therapy was achieved by inhibition of Notch1 and c-Myc mRNA and protein expression, which suppressed the Notch1/c-Myc signaling pathway.

Together these results suggest that the combined use of DHA and siNotch1 therapy for T-cell lymphoma is useful. The underlying mechanism of combined therapy is the effect on the expression of Notch1/c-Myc signaling pathway components. Thus, combining traditional Chinese medicine containing DHA with siRNA targeting the Notch1/c-Myc signaling pathway may represent a novel strategy for treating human T-cell lymphoma.

\section{Acknowledgements}

We thank Edanz Group (www.edanzediting.com/ac) for editing a draft of this study. This study was supported by Youth Funding of Affiliated Hospital, Qingdao University, Shandong, China (KZJ-34) and Science and Technology plan of Traditional Chinese Medicine Development Projects, Shandong, China (2017-197).

\section{References}

1. Taylor GP and Matsuoka M: Natural history of adult T-cell leukemia/lymphoma and approaches to therapy. Oncogene 24 : 6047-6057, 2005.

2. Sharma VM, Draheim KM and Kelliher MA: The Notch1/c-Myc pathway in T cell leukemia. Cell Cycle 6: 927-930, 2007.

3. Weng AP, Millholland JM, Yashiro-Ohtani Y, Arcangeli ML, Lau A, Wai C, Del Bianco C, Rodriguez CG, Sai H, Tobias J, et al: c-Myc is an important direct target of Notch1 in T-cell acute lymphoblastic leukemia/lymphoma. Gene Dev 20: 2096-2109, 2006.

4. Pear WS, Aster JC, Scott ML, Hasserjian RP, Soffer B, Sklar J and Baltimore D: Exclusive development of T cell neoplasms in mice transplanted with bone marrow expressing activated Notch alleles. J Exp Med 183: 2283-2291, 1996.

5. Klinakis A, Szabolcs M, Politi K, Kiaris H, Artavanis-Tsakonas S and Efstratiadis A: Myc is a Notch1 transcriptional target and a requisite for Notch1-induced mammary tumorigenesis in mice. Pro Natl Acad Sci USA 103: 9262-9267, 2006.

6. Rizzo P, Osipo C, Foreman K, Golde T, Osborne B and Miele L: Rational targeting of Notch signaling in cancer. Oncogene 27: 5124-5131, 2008.

7. Roy M, Pear WS and Aster JC: The multifaceted role of Notch in cancer. Curr Opin Genet Dev 17: 52-59, 2007.

8. Sharma VM, Calvo JA, Draheim KM, Cunningham LA, Hermance N, Beverly L, Krishnamoorthy V, Bhasin M, Capobianco AJ and Kelliher MA: Notch1 contributes to mouse $\mathrm{T}$-cell leukemia by directly inducing the expression of c-Myc. Mol Cell Biol 26: 8022-8031, 2006.

9. Kong R, Feng J, Ma Y, Zhou B, Li S, Zhang W, Jiang J, Zhang J, Qiao Z, Zhang T, et al: Silencing NACK by siRNA inhibits tumorigenesis in non-small cell lung cancer via targeting Notch1 signaling pathway. Oncol Rep 35: 2306-2314, 2016.

10. Li Y, Wang J, Li C and Ke XY: Contribution of PD-L1 to oncogenesis of lymphoma and its RNAi-based targeting therapy. Leuk Lymphoma 53: 2015-2023, 2012.

11. Yang Z, Qi Y, Lu C, Zhang J, Luo R and Kang S: Small interfering RNA (siRNA)-mediated knockdown of Notch1 suppresses tumor growth and enhances the effect of IL-2 immunotherapy in malignant melanoma. J BUON 20: 1553-1564, 2015.

12. Woo SH, Parker MH, Ploypradith P, Northrop J and Posner GH: Direct conversion of pyranose anomeric $\mathrm{OH} \rightarrow \mathrm{F} \rightarrow \mathrm{R}$ in the artemisinin family of antimalarial trioxanes. Tetrahedron Lett 39: 1533-1536, 1998.

13. Wang Q, Wu S, Zhao X, Zhao C, Zhao H and Huo L: Mechanisms of Dihydroartemisinin and Dihydroartemisinin/holotransferrin cytotoxicity in T-cell lymphoma cells. PLoS One 10: e0137331, 2015. 
14. Dong F, Tian H, Yan S, Li L, Dong X, Wang F, Li J, Li C, Cao Z Liu X and Liu J: Dihydroartemisinin inhibits endothelial cell proliferation through the suppression of the ERK signaling pathway. Int J Mol Med 35: 1381-1387, 2015.

15. Zhang CZ, Pan Y, Cao Y, Lai PB, Liu L, Chen GG and Yun J: Histone deacetylase inhibitors facilitate dihydroartemisinin-induced apoptosis in liver cancer in vitro and in vivo. PLoS One 7: 1726-1729, 2012

16. Wang SJ, Gao Y, Chen H, Kong R, Jiang HC, Pan SH, Xue DB, Bai XW and Sun B: Dihydroartemisinin inactivates NF-kappa B and potentiates the anti-tumor effect of gemcitabine on pancreatic cancer both in vitro and in vivo. Cancer Lett 293: 99-108, 2010.

17. Livak KJ and Schmittgen TD: Analysis of relative gene expression data using real-time quantitative PCR and the 2(-Delta Delta C(T)) method. Methods 25: 402-408, 2001.

18. Kamstrup MR, Biskup E, Gjerdrum LM, Ralfkiaer E, Niazi O and Gniadecki R: The importance of Notch signaling in peripheral T-cell lymphomas. Leuk lymph 55: 639-644, 2014.

19. Pear WS and Aster JC: T cell acute lymphoblastic leukemia/lymphoma: A human cancer commonly associated with aberrant NOTCH1 signaling. Curr Opin Hematol 11: 426-433, 2004

20. Lu JJ, Meng LH, Shankavaram UT, Zhu CH, Tong LJ, Chen G, Lin LP, Weinstein JN and Ding J: Dihydroartemisinin accelerates c-MYC oncoprotein degradation and induces apoptosis in c-MYC-overexpressing tumor cells. Biochem Pharmaco 80 22-30, 2010.

21. Sun HW, Wu C, Tan HY and Wang QS: Combination DLL4 with Jagged1-siRNA can enhance inhibition of the proliferation and invasiveness activity of human gastric carcinoma by Notch1/VEGF pathway. Hepatogastroenterology 59: 924-929, 2012.

22. Bin Hafeez B, Mustafa Adhami V, Asim M, Siddiqui IA, Bhat KM, Zhong W, Saleem M, Din M, Setaluri V and Mukhtar H: Targeted knockdown of Notch1 inhibits invasion of human prostate cancer cells concomitant with inhibition of matrix metalloproteinase- 9 and urokinase plasminogen activator. Clin Cancer Res 15: 452-459, 2009
23. Zhao X, Zhong H, Wang R, Liu D, Waxman S, Zhao L and Jing Y: Dihydroartemisinin and its derivative induce apoptosis in acute myeloid leukemia through Noxa-mediated pathway requiring iron and endoperoxide moiety. Oncotarget 6: 5582-5596, 2015.

24. He Q, Shi JX, Shen XL, An J, Sun H, Wang L, Hu YJ, Sun Q, Fu LC, Sheikh MS and Huang Y: Dihydroartemisinin upregulates death receptor 5 expression and cooperates with TRAIL to induce apoptosis in human prostate cancer cells. Cancer Biol Ther 9: 819-824, 2010.

25. Cabello CM, Lamore SD, Bair WB III, Qiao S, Azimian S, Lesson JL and Wondrak GT: The redox antimalarial dihydroartemisinin targets human metastatic melanoma cells but not primary melanocytes with induction of NOXA-dependent apoptosis. Invest New Drug 30: 1289-1301, 2012.

26. Liao K, Li J and Wang ZL: Dihydroartemisinin inhibits cell proliferation via AKT/GSK3 $3 /$ cyclinD1 pathway and induces apoptosis in A549 lung cancer cells. Int J Clin Exp Patho 7: 8684-8691, 2014.

27. Tong Y, Liu Y, Zheng H, Zheng L, Liu W, Wu J, Ou R, Zhang G, $\mathrm{Li} \mathrm{F}, \mathrm{Hu} \mathrm{M}$, et al: Artemisinin and its derivatives can significantly inhibit lung tumorigenesis and tumor metastasis through Wnt $/ \beta$-catenin signaling. Oncotarget 7: 31413-31428, 2016.

28. Handrick R, Ontikatze T, Bauer KD, Freier F, Rübel A, Dürig J, Belka C and Jendrossek V: Dihydroartemisinin induces apoptosis by a Bak-dependent intrinsic pathway. Mol Cancer Ther 9: 2497-2510, 2010.

29. Han P, Luan Y, Liu Y, Yu Z, Li J, Sun Z, Chen G and Cui B: Small interfering RNA targeting Racl sensitizes colon cancer to dihydroartemisinin-induced cell cycle arrest and inhibited cell migration by suppressing NFKB activity. Mol Cell Biochem 379: 171-180, 2013.

30. Kim MJ, Park JS, Lee SJ, Jang J, Park JS, Back SH, Bahn G, Park JH, Kang YM, Kim SH, et al: Notch1 targeting siRNA delivery nanoparticles for rheumatoid arthritis therapy. J Control Release 216: 140-148, 2015.

31. Tezgel AÖ, Gonzalez-Perez G, Telfer JC, Osborne BA, Minter LM and Tew GN: Novel protein transduction domain mimics as nonviral delivery vectors for siRNA targeting NOTCH1 in primary human T cells. Mol Ther 21: 201-209, 2013. 